\title{
Simple model of stacking-fault energies
}

\author{
Stokbro, Kurt; Jacobsen, Lærke Wedel
}

Published in:

Physical Review B

Link to article, DOI:

10.1103/PhysRevB.47.4916

Publication date:

1993

Document Version

Publisher's PDF, also known as Version of record

Link back to DTU Orbit

Citation (APA):

Stokbro, K., \& Jacobsen, L. W. (1993). Simple model of stacking-fault energies. Physical Review B, 47(9), 49164921. https://doi.org/10.1103/PhysRevB.47.4916

\section{General rights}

Copyright and moral rights for the publications made accessible in the public portal are retained by the authors and/or other copyright owners and it is a condition of accessing publications that users recognise and abide by the legal requirements associated with these rights.

- Users may download and print one copy of any publication from the public portal for the purpose of private study or research.

- You may not further distribute the material or use it for any profit-making activity or commercial gain

- You may freely distribute the URL identifying the publication in the public portal

If you believe that this document breaches copyright please contact us providing details, and we will remove access to the work immediately and investigate your claim 


\title{
Simple model of stacking-fault energies
}

\author{
K. Stokbro and K. W. Jacobsen \\ Laboratory of Applied Physics, Technical University of Denmark, DK 2800 Lyngby, Denmark
}

(Received 14 July 1992)

\begin{abstract}
A simple model for the energetics of stacking faults in fcc metals is constructed. The model contains third-nearest-neighbor pairwise interactions and a term involving the fourth moment of the electronic density of states. The model is in excellent agreement with recently published local-density calculations of stacking-fault energies, and gives a simple way of understanding the calculated energy contributions from the different atomic layers in the stacking-fault region. The two parameters in the model describe the relative energy contributions of the $s$ and $d$ electrons in the noble and transition metals, and thereby explain the pronounced differences in energetics in these two classes of metals. The model is discussed in the framework of the effective-medium theory where it is possible to find a functional form for the pair potential and relate the contribution associated with the fourth moment of the density of states with the so called one-electron correction energy.
\end{abstract}

\section{INTRODUCTION}

With the development of newer and faster computers it has become possible to make more realistic large-scale simulations of material properties at the atomic level. A crucial input to such simulations is an interatomic potential, which describes the essential physics of the system accurately. One of the most important physical processes when deforming materials is the emittance and interaction of dislocations. In most structures, the dislocations release energy by splitting into partial dislocations, thereby creating a stacking fault in between. The energy of the stacking fault will then determine the equilibrium distance between the partials. It is therefore an important feature of a potential to be able to calculate stacking-fault energies accurately. These are small energy differences, of the order $\mathrm{meV} /$ atom, due mainly to the changes in the band structure of the faulted crystal. It is a challenge to devise models for the energetics of stacking faults which are simple enough to be practical for simulations and yet accurate enough to describe these small energy differences reasonably well.

In recent years, a large number of simulations of metals and metal surfaces have been based on the effectivemedium theory, ${ }^{1}$ the embedded-atom method, ${ }^{2}$ or mathematically equivalent models like the $N$-body potentials ${ }^{3}$ or the glue model. ${ }^{4}$ These potentials contain, in addition to a pair potential, an embedding function $F\left(\bar{n}_{i}\right)$. This function gives the energy contribution as a function of the local-electron density $\bar{n}_{i}$, which is obtained by superimposing atomiclike densities $\rho(r)$ from the neighboring atoms, $\bar{n}_{i}=\sum_{j} \rho\left(r_{i j}\right)$. For a stacking fault in a fcc material, the distances to the first- and second-nearest neighbors are identical to those of the perfect crystal, so the change in the local-electron density will be minor. It is therefore a good approximation to replace the embedding function with its first-order expansion, $F\left(\bar{n}_{i}\right) \approx \frac{\partial F}{\partial \bar{n}_{i}} \bar{n}_{i}$, which is a pair potential. For the stacking faults the density-dependent term therefore effectively reduces to a pair potential.

By construction of a simple two-parameter model for the energetics of stacking-faults in fcc metals, we shall show that it is only for the noble metals that a centralforce model provides an appropriate description of the energetics in a stacking-fault region. For the transition metals and also for aluminum the difference in directional bonding between the stacking-fault region and the perfect crystal becomes important, and a central-force model is not sufficient. The directional nature of the bonding manifests itself in the electronic structure and can be described through an expansion of the electronic density of states in terms of its energy moments. This gives rise to effective four-body interactions which have to be taken into account. The two parameters in the model therefore describe the strengths of the pairwise interactions and the four-body interactions.

The model is tested by comparison with recent localdensity calculations of stacking-fault energies for eight fcc metals: ${ }^{5,6} \mathrm{Cu}, \mathrm{Ag}, \mathrm{Au}, \mathrm{Ni}, \mathrm{Pd}, \mathrm{Rh}, \mathrm{Ir}$, and Al. The calculations $^{5,6}$ give the contributions to the extrinsic, intrinsic, and twin stacking faults projected onto individual atomic layers. The comparison shows that the simple model can describe the energies of the different stacking faults as well as the-variation in the layer-projected energy through each of the stacking faults.

In Sec. II we present the model for the stacking-fault energetics, and thereafter in Sec. III we compare the model to the results of the above-mentioned $a b$ initio calculations. Section IV provides a derivation of the model from the effective-medium theory and presents an estimate of the four-body terms based on a tight-binding linear muffin-tin orbital (LMTO) Hamiltonian. In Sec. V we conclude.

\section{THE MODEL}

In the model, there are two contributions to the stacking-fault energy $E$. The first term $E_{\text {pair }}$ is a sum 
of pairwise interactions between the atoms while the second term $E_{\text {band }}$ is obtained from the electronic density of states:

$$
E=E_{\text {pair }}+E_{\text {band }} \text {. }
$$

The guiding line in the construction of the model is to keep it as simple as possible with only a minimal input.

In a stacking-fault region the fcc-stacking sequence $A B C$ is changed to hcp stacking $A B A$. The stacking sequences for the extrinsic, intrinsic, and twin stacking faults, that we shall discuss here, are indicated in Fig. 1. The change in stacking sequence does not change the very close environment around an atom. Every atom in a stacking-fault region is still surrounded by 12 nearest neighbors and 6 second-nearest neighbors and since the interlayer relaxation is unimportant for stacking faults, ${ }^{7}$ we can assume that these distances are the same as in the perfect fcc crystal. A pair potential with a range only extending to second-nearest-neighbor distances does, therefore, not give any contribution to the energy of a stacking fault. However, if the pair potential is sufficiently long ranged an energy difference between the hcp and fcc stackings will contribute at the third-nearest-neighbor level and beyond. If we, for simplicity, truncate the pair potential $\phi(r)$ after the fifth-nearest-neighbor shell, there will be a specific contribution to the stacking-fault energy from the atoms which are in a hcp-stacking sequence. To be more precise: There will be an energy contribution $e_{2}$ to the stacking-fault energy each time two layers of the same kind $(A, B$, or $C)$ are separated by only one other layer. The energy $e_{2}$ is given in terms of the pair potential by
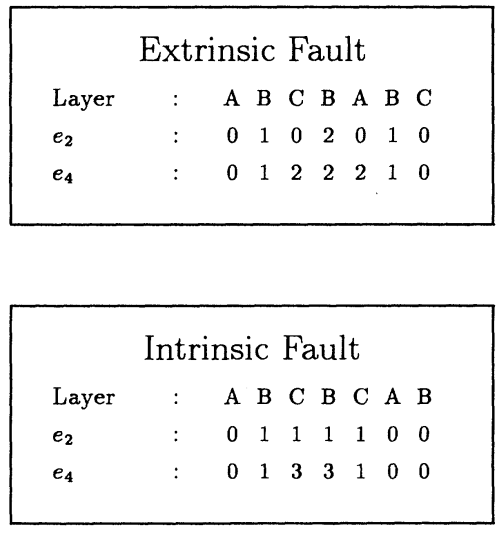

\begin{tabular}{|lllllllllll}
\hline \multicolumn{8}{c|}{ Twin Fault } \\
Layer & $:$ & A & B & C & B & A & C & B \\
$e_{2}$ & $:$ & 0 & 1 & 0 & 1 & 0 & 0 & 0 \\
$e_{4}$ & $:$ & 0 & 1 & 2 & 1 & 0 & 0 & 0 \\
& &
\end{tabular}

FIG. 1. Layer-by-layer contributions to the extrinsic, intrinsic, and twin stacking-fault energies in units of the parameters $e_{2}$ (from the pairwise interactions) and $e_{4}$ (from the fourth moment of the density of states).

$$
\begin{aligned}
e_{2}= & \phi\left(\sqrt{\frac{8}{3}}\right)-3 \phi(\sqrt{3})+6 \phi\left(\sqrt{\frac{11}{3}}\right) \\
& -3 \phi(\sqrt{4})-6 \phi(\sqrt{5})+6 \phi\left(\sqrt{\frac{17}{3}}\right)
\end{aligned}
$$

where we have explicitly given the changed neighbor distances in units of the nearest-neighbor distance. ${ }^{8}$

The contribution to the stacking-fault energy from the pairwise interactions gives rise to a particular pattern when decomposed into contributions from each layer in the stacking fault. In Fig. 1 is indicated the number of $e_{2}$ contributions for each layer in the extrinsic, intrinsic, and twin stacking faults. This projection onto the individual layers will be useful when the model is compared with results from local-density calculations.

The second contribution to the energy of the stacking faults comes from the shape of the electronic density of states. To describe the electronic structure in the metal, we consider a tight-binding model with Hamiltonian $H$ which only contains hopping-matrix elements between nearest neighbors. (The construction of the tight-binding model will be discussed in greater detail in Sec. IV.) We shall assume that the atomic levels and hopping matrix elements are the same in a stacking-fault region as in a perfect fcc crystal. The aim is to find a simple way of estimating the band energy $E_{\text {band }}$ which is given by the density of states (projected onto state $i$ ) $\rho_{i}(\epsilon)$ by

$$
E_{\mathrm{band}}=\int_{-\infty}^{\epsilon_{F}} \epsilon \rho_{i}(\epsilon) d \epsilon
$$

where $\epsilon_{F}$ denotes the Fermi energy. To this end, we characterize the projected density of states by its moments $\mu_{n}^{i}=\int_{-\infty}^{\infty} \epsilon^{n} \rho_{i}(\epsilon) d \epsilon$. The moments have a geometrical interpretation since they can be found by traversing the lattice,

$\mu_{n}^{i}=\left\langle i\left|H^{n}\right| i\right\rangle=\sum_{j, k, l, \ldots}\langle i|H| j\rangle\langle j|H| k\rangle\langle k|\cdots| l\rangle\langle l|H| i\rangle$.

The calculation of the $n$th moment requires a summation over all paths in the lattice with $n$ legs. The band energy is in principle a function of all the moments, but it is often possible to get estimates of energy changes by considering only the changes in a few moments. (For a general discussion of this approach the reader is referred to Carlsson. ${ }^{9}$ ) We first note that if we restrict the model to nearest-neighbor hopping, the moments up to third order will be the same in a stacking-fault region as in a perfect fcc crystal. The first nonzero contribution therefore appears in the fourth moment, and for simplicity we shall only include this term here. (See Sec. IV for a discussion of the higher-order moments.) The difference between the fourth moment of the density of states of an atom in a stacking-fault region and an atom in a perfect fcc crystal is quite small and it is therefore reasonable to use a linear expansion of the change in band energy

$$
\Delta E_{\mathrm{band}}=\sum_{i} \alpha_{4}\left(\mu_{4}^{i}-\mu_{4, \mathrm{fcc}}^{i}\right)
$$

The fourth moment $\mu_{4}^{i}$ contains information about the 
environment of atom $i$ which can be reached by closed paths with four legs. The paths can be divided into two classes: Ring paths and single-track paths. The singletrack paths use the same two legs out and back, while the ring paths visit each atom in the paths only once. The closed paths with four legs can extend over at most three layers in the crystal and we therefore now consider the two different situations where three layers are in a fcc$(A B C)$ stacking sequence and in a hcp- $(A B A)$ stacking sequence. All the paths which only visit two layers of the fcc-stacking sequence have a counterpart in the hcpstacking sequence so there can be no contribution to the stacking-fault energies from these paths. Consider first the paths which start at an atom in the $A$ layer. In both situations there are nine single-track paths and six ring paths which visit all three layers starting at $A$. In an $s$ band model where there is no angular dependence of the hopping amplitudes, all the paths contribute with the same amount, and there will be no net contribution to the stacking-fault energy from these paths. However, if we consider $p$ or $d$ states, the paths are no longer equivalent, and there will be a contribution to the stacking-fault energy which we denote by $e_{4}$.

Let us now consider the paths which start at the central $B$ layer. For both the fcc- and the hcp-stacking sequences there are 18 single-track paths and 12 ring paths, and there is an exact two-to-one correspondence between these roads and the roads starting at layer $A$. (This is easily seen from Fig. 2.) Therefore, there is an energy

(a)

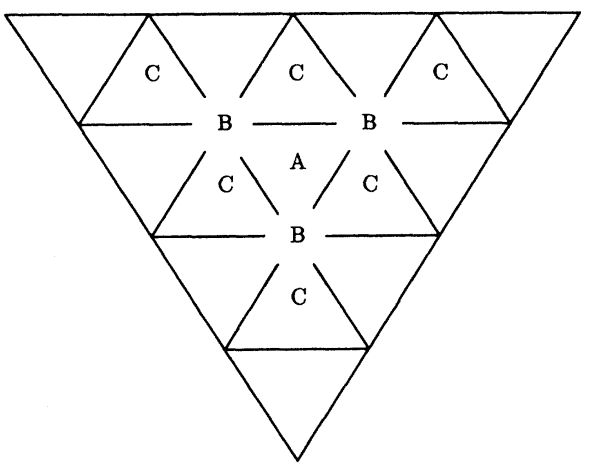

(b)

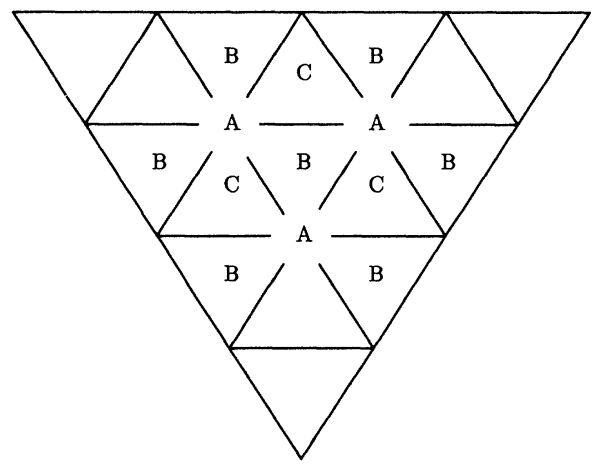

FIG. 2. The atoms visited by roads with four legs which traverse all three planes in an $A B C$ sequence. (a) The roads start in the $A$ layer. (b) The roads start at the central atom in the $B$ layer. contribution from the central layer of the hcp-stacking sequence of $2 e_{4}$.

Using this approach, it is possible to find the layerwise contribution to the extrinsic, intrinsic, and twin stacking faults from the fourth-moment term in units of $e_{4}$. The result is shown in Fig. 1.

\section{TESTING THE MODEL}

We will now test our model on the data in Ref. 5, where calculated results are presented for layer-by-layer energy contributions to the extrinsic, intrinsic, and twin faults for eight different metals. ${ }^{10}$ The calculations are performed using the layer Korringer-Kohn-Rostoker method together with the force theorem. To see if the layer decompositions of the stacking faults obtained from this local-density calculation follow that of Fig. 1, we have fitted the two parameters $e_{2}$ and $e_{4}$ to their data; that is, a two-parameter fit to 12 data points for each element. The goodness of the fits and the fitted values $\left(e_{2}^{\mathrm{fit}}\right.$ and $e_{4}^{\text {fit }}$ ) are shown in Table I, while Fig. 3 shows the results

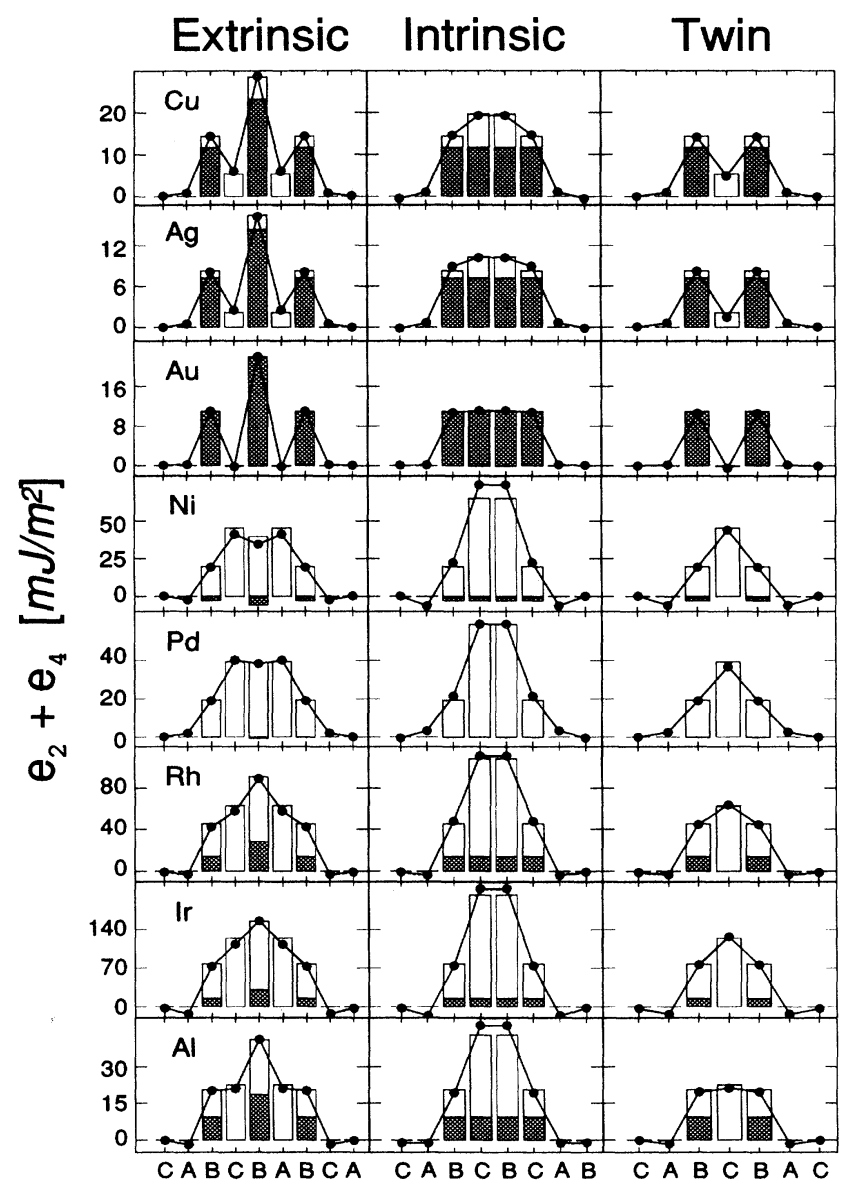

FIG. 3. Layer-by-layer energy contributions to the extrinsic, intrinsic, and twin faults. The solid lines are the results of the local-density calculations in Ref. 5 . The bars are the energy profiles obtained from Fig. 1, where the solid part is the contribution from $e_{2}$ and the open part the contribution from $e_{4}$. 
TABLE I. The result of fitting the layer structure in Fig. 1 to the data of Ref. 5. The goodness of the fits is given by $\left\langle\left(E-E^{\mathrm{fit}}\right)^{2}\right\rangle^{\frac{1}{2}} /\left\langle E^{2}\right\rangle^{\frac{1}{2}}$, with the average taken over the 12 data points used in each fit. Also shown are the values of $e_{4}$ obtained from a LMTO calculation.

\begin{tabular}{lcrrrrrrrr}
\hline \hline & Unit & \multicolumn{1}{c}{$\mathrm{Cu}$} & $\mathrm{Ag}$ & \multicolumn{1}{c}{$\mathrm{Au}$} & $\mathrm{Ni}$ & $\mathrm{Pd}$ & \multicolumn{1}{c}{$\mathrm{Rh}$} & \multicolumn{1}{c}{$\mathrm{Ir}$} & \multicolumn{1}{c}{$\mathrm{Al}$} \\
\hline Goodness & $(\%)$ & 2.3 & 4.3 & 2.2 & 11.2 & 3.3 & 4.5 & 5.7 & 6.7 \\
$e_{2}^{\text {fit }}$ & $\left(\mathrm{mJ} / \mathrm{m}^{2}\right)$ & 11.6 & 7.2 & 11.0 & -3.0 & -0.3 & 14.1 & 15.2 & 9.3 \\
$e_{4}^{\text {fit }}$ & $\left(\mathrm{mJ} / \mathrm{m}^{2}\right)$ & 2.7 & 1.0 & -0.0 & 22.6 & 19.6 & 31.4 & 62.2 & 11.2 \\
\hline$e_{4}^{\text {calc }}$ & $\left(\mathrm{mJ} / \mathrm{m}^{2}\right)$ & 0.0 & 0.0 & 0.0 & 18.7 & 21.2 & 30.4 & 37.3 & 5.0 \\
\hline \hline
\end{tabular}

graphically. The complicated energy profiles of the stacking faults are generally very well described by this simple model.

The metals clearly fall into two groups. The stacking faults of the noble metals are described almost exclusively by the pairwise interaction term $e_{2}$ while the transition metals have much larger stacking-fault energies which must be attributed to the band (four-body) term $e_{4}$. Aluminum seems to be an intermediate between the two. It was noted above that a pure $s$-band cannot contribute to the fourth-body term and, of course, a filled $d$ band as for the noble metals cannot contribute either. The pairwise interaction therefore dominates for the noble metals. For the transition metals, however, the partly filled $d$ band gives a strong contribution to the fourth-moment term which then completely dominates the pairwise interactions.

Our model assigns an energy of $2 e_{2}+4 e_{4}$ to each hcpstacking sequence $(A B A)$. There are two of these in the extrinsic and the intrinsic faults, but only one in the twin fault. Our model therefore predicts the following relation between the stacking-fault energies:

$$
\frac{1}{2} E_{\mathrm{intr}}=\frac{1}{2} E_{\mathrm{extr}}=E_{\mathrm{twin}}=E_{\mathrm{hcp}}=2 e_{2}+4 e_{4} .
$$

In Table II we compare the total energies of these faults. Apart from the hcp-structural energies, which have been calculated with a different method, the relationships hold within a few percent. However, there is a tendency for the transition metals that the energy of the intrinsic fault is a little higher than the energy of the extrinsic and twin faults. The origin of this difference is that in the intrinsic fault the hcp-stacking sequences are not separated, so that there is an interaction energy between the two $A B A$ sequences. Such interactions are neglected in our model, which has a range of only two layers.

TABLE II. The total energy predicted by our model $\left(2 e_{2}^{\mathrm{fit}}+4 e_{4}^{\mathrm{fit}}\right)$ compared with local-density calculations of stacking faults (Ref. 5) and the hcp structure (Refs. 17 and 7). The unit is $\mathrm{mJ} / \mathrm{m}^{2}$.

\begin{tabular}{lcccccccc}
\hline \hline & $\mathrm{Cu}$ & $\mathrm{Ag}$ & $\mathrm{Au}$ & $\mathrm{Ni}$ & $\mathrm{Pd}$ & $\mathrm{Rh}$ & $\mathrm{Ir}$ & $\mathrm{Al}$ \\
\hline $2 e_{2}^{\text {fit }}+4 e_{4}^{\text {fit }}$ & 34 & 18 & 22 & 84 & 78 & 154 & 279 & 63 \\
$\frac{1}{2} E_{\text {extr }}$ & 37 & 19 & 22 & 75 & 78 & 141 & 247 & 59 \\
$\frac{1}{2} E_{\text {intr }}$ & 35 & 17 & 22 & 90 & 81 & 154 & 267 & 62 \\
$E_{\text {twin }}$ & 36 & 19 & 21 & 70 & 76 & 147 & 243 & 56 \\
$E_{\text {hcp }}$ & 30 & 15 & 28 & 103 & 121 & 164 & 281 & 85 \\
\hline \hline
\end{tabular}

\section{CONNECTION TO THE EFFECTIVE-MEDIUM THEORY}

The simple two-parameter model for the stacking-fault energetics can be understood from other models of interatomic interactions. In the tight-binding approach, ${ }^{11}$ the total energy is written as a sum of pair potentials plus the contribution from a tight-binding Hamiltonian; so the simple model follows right away if the hopping amplitudes only extend to nearest neighbors and the density of states is expanded in terms of moments.

In the effective-medium theory ${ }^{1}$ an embedding density $\bar{n}_{i}$ is associated with each atom. The embedding density is calculated by averaging over the electron-density tails of neighboring atoms. The total energy is then calculated by moving each atom to a reference system, taken to be a fcc crystal with the same embedding density, and evaluate the energy difference. The binding energy of atom $i, E_{b}(i)$, is then given by three terms

$$
E_{b}(i)=E_{c}\left(\bar{n}_{i}\right)+E_{\mathrm{AS}}(i)+E_{\mathrm{el}}(i) .
$$

The first term the cohesive function $E_{c}$ is the energy of the reference system. The two last terms are the change in energy when the atom is moved to the reference system. The atomic-sphere correction $E_{\mathrm{AS}}$ is the change in electrostatic and exchange-correlation energy and is usually represented as a difference between a pair potential contribution in the real system and in the reference system. The one-electron-correction energy $E_{\mathrm{el}}$ is the change in the sum over the one-electron eigenvalues, i.e., it is the difference in band energy between the real system and the reference system where the potential has to be shifted rigidly from the reference system to the real system.

The changes of the embedding densities in a stackingfault region are very small and it is therefore possible to linearize the density-dependent terms $E_{c}$ and $E_{\mathrm{AS}}$ whereby an effective pair potential $\phi(r)$ results. This pair potential is given by

$\phi(r)=\frac{\alpha n_{0}}{12}\left(\frac{\eta}{\gamma_{1}\left(\eta+\eta_{1}\right)} e^{-\eta_{2}(r-1) \beta s_{0}}-\frac{1}{\gamma_{2}} e^{-\eta(r-1) s_{0}}\right)$,

in terms of the effective-medium parameters $\eta, \eta_{1}, \eta_{2}, \gamma_{1}, \gamma_{2}, \alpha n_{0}$, the Wigner-Seitz radius $s_{0}$, and the distance $r$ in units of the nearest-neighbor distance $\beta s_{0}$. Using these parameters, we can now in principle calculate $e_{2}$ from Eq. (2). However, Fig. 4 shows the value 


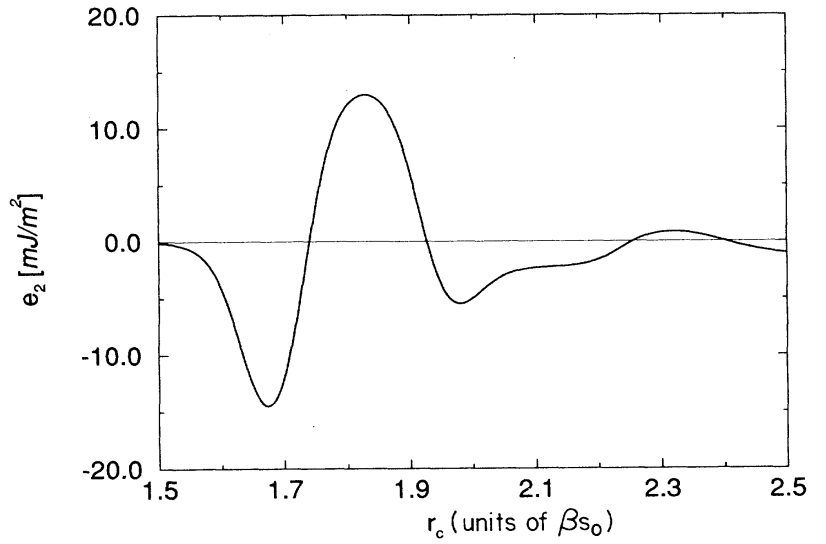

FIG. 4. The value of $e_{2}$ for copper obtained from Eq. (2), where the pair potential $\phi$ is cut off softly with a Fermi function, $\left(1+e^{40\left(r-r_{c}\right)}\right)^{-1}$. The figure shows the value of $e_{2}$ as a function of the position of the cutoff $r_{c}$.

of $e_{2}$ for copper for different cutoffs of the pair potential $\phi$. The result oscillates ${ }^{12}$ and can take any value in an interval of the same order as $e_{2}^{\text {fit }}$ dependent on the cutoff; this curve is characteristic for all the elements. The reason is simply that the effective-medium assumption of an exponential tail of the density breaks down outside the first two neighbor shells, probably because the Friedel oscillations then begin to be important. From a pragmatic point of view, however, we can easily construct a good empirical pair potential ${ }^{12}$ by choosing a value of $r_{c}$, such that we obtain the fitted value $e_{2}^{\text {fit }}$. For copper this value is $r_{c}=1.8$, which is between the third- (distance $\sqrt{3}$ ) and fourth- (distance $\sqrt{4}$ ) neighbor shells. The position of the cutoff will not affect most other physical properties calculated with the potential, because it is only for an atom in a stacking-fault region that the distances to first- and second-nearest neighbors are unchanged compared to the reference system. For other configurations the energy will therefore be dominated by contributions from the first- and second-nearest-neighbor shells and the long-range part of the pair potential will be unimportant.

The fourth-moment contribution $e_{4}$ is contained in the one-electron energy and we shall in the following calculate this term using the recursion method. By controlled approximations, we will simplify this to an expansion in the fourth moment only. First of all, we estimate the difference in band energy between the stacking-fault region and a perfect fcc crystal by use of a tight-binding Hamiltonian, which we construct from a first-order LMTO approximation

$H_{R L, R^{\prime} L^{\prime}}^{\alpha}=C_{R L}^{\alpha} \delta_{R R^{\prime}} \delta_{L L^{\prime}}+\left(\Delta_{R L}^{\alpha}\right)^{1 / 2} S_{R L, R^{\prime} L^{\prime}}^{\alpha}\left(\Delta_{R^{\prime} L^{\prime}}^{\alpha}\right)^{1 / 2}$

in the localized representation $\alpha_{\mathrm{I}}$ ( $s p$ screening) or $\alpha_{\mathrm{II}}$ (spd screening). ${ }^{13}$ The off-site elements of the structure constants we calculate from the interpolation formulas in Ref. 13, including only nearest-neighbor hops. We use the same on-site elements on all sites, which we take to be the fcc-bulk value. These we calculate by inserting the off-site elements into the LMTO "Dysons equation" for the structure constants. For the potential parameters $(C, \Delta)$ we use the fcc-equilibrium values listed in Ref. 14 transformed to the relevant representation.

From this Hamiltonian, we get the projected density of states using the recursion method. ${ }^{15}$ This gives the Greens function as an infinite-fraction expansion in the chain parameters $a_{n}$ and $b_{n}$,

$$
G(\epsilon)=\frac{1}{\epsilon-a_{0}+\frac{b_{1}^{2}}{\epsilon-a_{1}+\frac{b_{2}^{2}}{\vdots}}} .
$$

We will terminate the expansion with a constant chain after the $n$th level, which gives rise to a square-root terminator

$$
T(\epsilon)=\frac{\epsilon-a_{\infty}}{2}-i \sqrt{b_{\infty}^{2}-\left(\frac{\epsilon-a_{\infty}}{2}\right)^{2}},
$$

resulting in a continuum of states in the range of $a_{\infty}-$ $2 b_{\infty}<\epsilon<a_{\infty}+2 b_{\infty}$. The constant chain is therefore determined by the top $\left(\epsilon_{T}\right)$ and the bottom $\left(\epsilon_{B}\right)$ of the band

$$
\begin{aligned}
& a_{\infty}=\frac{1}{2}\left(\epsilon_{T}+\epsilon_{B}\right), \\
& b_{\infty}=\frac{1}{4}\left(\epsilon_{T}-\epsilon_{B}\right) .
\end{aligned}
$$

In a solid the band edges, and thereby the limit of the recursion chain, will be determined by the extendedbulk states, and the chain will converge as $1 / n^{2}$ towards this limit in a three-dimensional (3D) ordered system. ${ }^{16}$ The first chain parameters $a_{0}, a_{1}, b_{1}$ are unchanged in the stacking-fault region compared to the fcc-bulk values. The next parameter, $b_{2}$, we calculate from the value of the fourth moment $\mu_{4}$, and the rest of the chain we obtain by interpolating between the second-level values $a_{1}, b_{2}$ and the fcc-bulk values using the $1 / n^{2}$ form. In this way, we get the one-electron correction as a function of the fourth moment. By linearizing we obtain the form used in Sec. II,

$$
E_{\mathrm{el}}=\alpha_{4}\left(\mu_{4}-\mu_{4}^{\mathrm{ref}}\right)
$$

however we now have a prescription for calculating $\alpha_{4}$.

To test the approach, we have calculated the structural-energy difference between fcc and hcp within a canonical $d$-band model. The results are shown in Fig. 5. The solid line indicates the energy as calculated from the recursion method using a chain of length 8 , while the dotted line is obtained from Eq. (14). Also shown is the result from a fully self-consistent calculation for the $3 d$, $4 d$, and $5 d$ transition metals. ${ }^{17}$ For comparison all the numbers have been rescaled with the potential parameter $\Delta_{d}^{\alpha_{I I}}$. The figure shows that for the elements with an almost filled $d$ shell, i.e., the elements with 8,9 , or $10 d$ electrons, the fourth-moment term gives a reasonable description. This is where we find the fcc metals and therefore the elements we have been considering in this work. If we instead consider elements in the first or middle part of the transition series, we cannot expect 


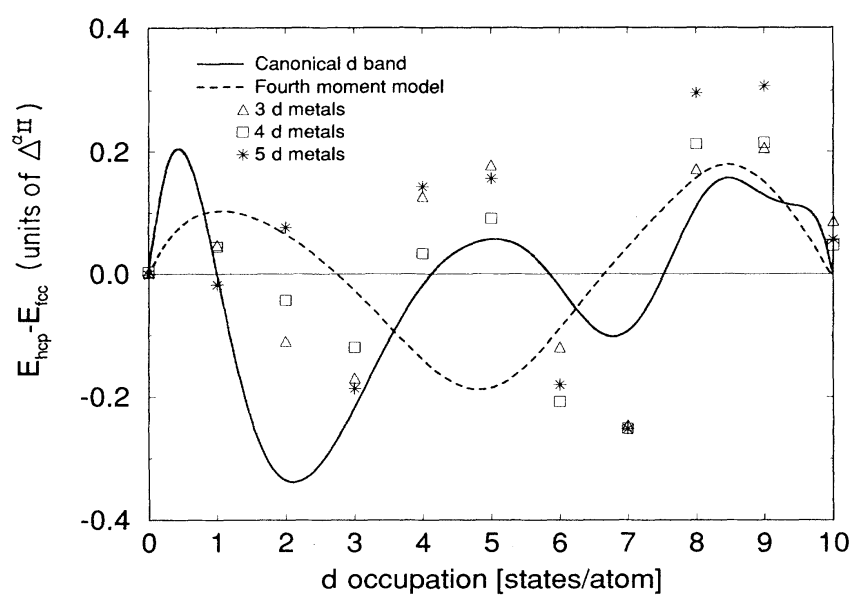

FIG. 5. The structural energy difference between hcp and fcc for the transition metals rescaled by the potential parameter $\Delta_{d}^{\alpha_{I I}}$. The triangles, squares, and stars are the results of a full LMTO calculation for the $3 d, 4 d$, and $5 d$ transition metals, respectively (Ref. 17). The solid line is the result of a canonical $d$-band model in the $\alpha_{\text {II }}$ representation, and the dotted line is the result when only the fourth moment is included.

the layer-by-layer contribution to be described only from a fourth-moment term.

With a similar calculation ${ }^{18}$ to that in Fig. 5 we have determined the value of the fourth-moment term $e_{4}^{\text {calc }}$ of the stacking faults. The results are shown in Table I and except for iridium and aluminum there is good agreement with the values obtained by fitting to the data of Ref. 5 . We beleive the discrepancy for rhodium arise from neglect of the $s$ electrons, since from Fig. 5 the large differ- ence in structural energy between iridium and rhodium can simply not be explained in a canonical $d$-band model.

\section{CONCLUSIONS}

We have discussed a simple two-parameter model for the layer-by-layer contributions to the stacking-fault energies, which explains the energy profiles found in recent $a b$ initio calculations. ${ }^{5}$ The two parameters can be interpreted as the relative contributions to the fault energies of the $s$ and $d$ electrons for the noble and transition metals, consistent with the observed difference in stackingfault energies for the two kinds of metals.

By well-defined approximations, we have put the oneelectron correction into a form involving only the fourth moment of the density of states. By comparisons with the fcc-hcp structural-energy difference, we have found this approximation to be justified for the metals with 8,9 , or $10 d$ electrons. Approximate calculations within the LMTO formalism of the fourth-moment term showed good agreement with the values obtained by fitting to the stacking-fault energies of Ref. 5 .

We have also showed that, by using a proper cutoff of the pair potentials in the effective-medium theory, we can get the correct value for the atomic-sphere correction energy. This indicates that the effective-medium theory, including the one-electron correction in the fourthmoment approximation, may provide a useful description of stacking-fault energies appropriate for large-scale simulations of material properties in fcc metals.

\section{ACKNOWLEDGMENTS}

We are grateful to H. L. Skriver, N. M. Rosengaard, B. Hammer, and S. Crampin, who have provided us with data from their calculations. This work was supported by the Danish Research Councils through the Center for Surface Reactivity.
${ }^{1}$ K.W. Jacobsen, J.K. Nørskov, and M.J. Puska, Phys. Rev. B 35 (14), 7423 (1987); K. W. Jacobsen, Comments Condens. Matter. Phys. 14, 129 (1988).

${ }^{2}$ M.S. Daw and M.I. Baskes, Phys. Rev. Lett. 50, 1285 (1983).

${ }^{3}$ M.W. Finnis and J.E. Sinclair, Philos. Mag. A 50, 45 (1984).

${ }^{4}$ F. Ercolessi, E. Tosatti, and M. Parinello, Phys. Rev. Lett. 57, 719 (1986).

${ }^{5}$ S. Crampin, K. Hampel, J.M. MacLaren, and D.D. Vvedensky, J. Mater. Res. 5, 2107 (1990).

${ }^{6}$ J. M. MacLaren, S. Crampin, D. D. Vvedensky, and J. B. Pendry, Phys. Rev. B 40, 12164 (1989); J. M. MacLaren, S. Crampin, D. D. Vvedensky, and M. E. Eberhart, Phys. Rev. Lett. 63, 2586 (1989).

${ }^{7}$ B. Hammer, K. W. Jacobsen, V. Milman, and M. C. Payne, J. Phys. Condens. Matter 4, 10453 (1992).

${ }^{8} \mathrm{~J}$. P. Hirth and J. Lothe, Theory of Dislocations (Wiley Interscience, New York, 1982).

${ }^{9}$ A.E. Carlsson, Solid State Phys. 43, 1 (1990).

${ }^{10}$ In Ref. 5 is published the layer-by-layer contribution of the one-electron energy to the stacking faults together with the total Madelung energy. Since the Madelung energy is calculated from a pair potential, we may decompose the energy according to Fig. 1, and thereby obtain the layer-by-layer decomposition of the total energy.

${ }^{11}$ For a review, see M. W. Finnis, A. T. Paxton, D. G. Pettifor, A. P. Sutton, and Y. Ohta, Philos. Mag. A 58, 143 (1988).

${ }^{12}$ H. Häkkinene, S. Mäkinen, and M. Manninen, Phys. Rev. B 41, 12441 (1990).

${ }^{13}$ H. J. Nowak, O. K. Andersen, T. Fujiwara, O. Jepsen, and P. Vargas, Phys. Rev. B 44, 3577 (1991).

${ }^{14}$ O.K. Andersen, O. Jepsen, and D. Glötzel, in Highlights of Condensed-Matter Theory, edited by F. Bassani, F. Fumi, and M. P. Tosi (North-Holland, New York, 1985).

${ }^{15}$ R. Haydock, V. Heine, and M.J. Kelly, J. Phys. C 8, 2591 (1975).

${ }^{16}$ R. Haydock, Philos. Mag. 37, 97 (1978).

${ }^{17}$ H.L. Skriver, Phys. Rev. B 31, 1909 (1985).

${ }^{18}$ For aluminum we use an $s p$ model similar to the one described in: N. Chetty, K. Stokbro, K. W. Jacobsen, and J. K. Nørskov, Phys. Rev. B 46, 3798 (1992). 